\title{
Caracterização física, química e sensorial de cookies confeccionados com Farinha de Talo de Couve (FTC) e Farinha de Talo de Espinafre (FTE) ricas em fibra alimentar
}

\author{
Phisical, chemical, and sensorial characterization of cookies made with \\ Kale Stalk Flour (KSF) and Spinach Stalk Flour (SSF) rich in nourishing fiber
}

\author{
Ana Karina MAURO ${ }^{1 *}$, Vera Lúcia Mathias da SILVA ${ }^{1}$, Maria Cristina Jesus FREITAS ${ }^{1}$
}

\begin{abstract}
Resumo
Objetivou-se utilizar Farinhas de Talos (FT) na confecção de cookies. As farinhas foram obtidas a partir de talos de couve manteiga e espinafre desidratados. Confeccionaram-se 3 tipos de cookies por modificação da formulação padrão de sugar-sanap cookie, método 10-50D (AMERICAN..., 1995): cookie controle, cookie com 15\% de FTC e com 15\% de FTE. Realizou-se a composição centesimal nas FT segundo as metodologias descritas da AOAC (ASSOCIATION..., 1995), sendo as fibras alimentares analisadas pelo método de van Soest (1963). Nos cookies, procedeu-se às seguintes análises: peso, espessura, diâmetro, volume e densidade aparente segundo método 10-50D da AACC (AMERICAN..., 1995), acidez titulável e pH através da técnica do Instituto Adolfo Lutz (SÃO PAULO, 1985), rendimento e fator térmico. A composição centesimal dos cookies seguiu normas da AOAC (ASSOCIATION..., 1995) e, para aceitação dos experimentais, utilizou-se escala hedônica de 9 pontos. Constatou-se que as FT possuem baixa densidade energética e alto teor de fibra alimentar e cinzas. Os cookies experimentais apresentaram maior $(\mathrm{p}<0,05)$ espessura, maior valor $(\mathrm{p}<0,05)$ para a acidez titulável e menor $(\mathrm{p}<0,05)$ valor para o $\mathrm{pH}$, quando comparados ao controle. A composição centesimal revelou menor teor de gordura e densidade calórica e maior teor de umidade e fibra nos cookies experimentais. A aceitação dos cookies foi considerada satisfatória, destacando-se o cookie contendo FTE.
\end{abstract}

Palavras-chave: cookies; farinha de talos; couve; espinafre.

\begin{abstract}
The objective of the study was to use Stalk Flours (SF) to prepare cookies. The ST was made with dehydrated Kale Stalks (KSF) and Spinach Stalks (SSF). Three kinds of cookies were prepared by modification of the sugar-snap cookie formula, 10-50D method (AMERICAN..., 1995): control cookie, cookie with $15 \%$ of KSF, and cookie with 15\% of SSF. The centesimal composition was performed on the SF by the AOAC (ASSOCIATION..., 1995) and the nourishing fibers were prepared according to van Soest (1963). The following parameters were analyzed: weight, thickness, diameter, volume, and apparent density according to the 10-50D method of the AACC (AMERICAN..., 1995), titratable acidity and $\mathrm{pH}$ using the Adolfo Lutz Institute (2005) technique, and also revenue and caloric factor. The determination of the centesimal composition of the cookies followed the AOAC regulations (ASSOCIATION..., 1995), and a 9-point hedonic scale of was used for the acceptance testing. It was observed that the SF had a low energetic density and a high value of nourishing fiber and ashes. The cookies analyzed showed a higher value of thickness $(\mathrm{p}<0,05)$, higher value $(\mathrm{p}<0,05)$ of titratable acidity, and a lower value $(\mathrm{p}<0,05)$ of $\mathrm{pH}$ compared to the control. The centesimal composition showed a lower value of fat and caloric density and a higher value of humidity and fiber in the cookies tested. The acceptance of the cookies was satisfactory, especially that with SSF.
\end{abstract}

Keywords: cookies; stalks flours; kale; spinach.

\section{Introdução}

A preocupação com a manutenção da saúde e com a prevenção de certas doenças tem sido associada a uma ingestão adequada de Fibra Alimentar (FA) por parte dos profissionais de saúde, e também por uma parcela da população atenta às informações que, já há algum tempo, vêm sendo veiculadas. A FA, considerada o principal componente de vegetais, frutas e cereais integrais, permitiu que estes alimentos pudessem ser incluídos na categoria dos alimentos funcionais, pois a sua utilização dentro de uma dieta equilibrada pode reduzir o risco de algumas doenças, como as coronarianas e certos tipos de câncer (FOOD..., 1998).
Além dos alimentos convencionais ricos em fibras, existem inúmeros alimentos não convencionais geralmente não utilizados na alimentação humana que possuem grande quantidade desse nutriente. Os resíduos de alimentos minimamente processados e os resíduos de frutas e hortaliças utilizadas na indústria alimentícia são, geralmente, desprezados e poderiam ser utilizados como fonte alternativa de fibras (PEREIRA, et al., 2003). A obtenção destes produtos é responsável pela produção de quantidades significativas de talos de hortaliças, eliminados durante as operações de seleção e corte, e que constituem grande desperdício para a indústria. Esses talos,

Recebido para publicação em 31/7/2008

Aceito para publicação em 20/6/2009 (003638)

Centro de Ciências da Saúde, Instituto de Nutrição Josué de Castro, Universidade Federal do Rio de Janeiro - UFRJ, Rua Souza Lima, n. 363, Ap. 308, CEP 22081-010,

Copacabana, Rio de Janeiro - RJ, Brasil, E-mail: kakamauro@yahoo.com.br

${ }^{*}$ A quem a correspondência deve ser enviada 
especificamente, possuem teores apreciáveis de fibra alimentar, e seu aproveitamento na elaboração de alimentos processados contribuirá para o aumento dos teores de fibra insolúvel na dieta, além de reduzir o acúmulo crescente dos desperdícios industriais (SÁNCHEZ; PÉREZ, 2001; PENNA; TUDESCA, 2001).

A FA pode ser utilizada no enriquecimento de produtos ou como ingrediente, pois é constituída de polissacarídeos, lignina, oligossacarídeos resistentes e amido resistente, entre outros, que apresentam diferentes propriedades físico-químicas. De maneira geral, estas propriedades permitem inúmeras aplicações na indústria de alimentos, substituindo gordura ou atuando como agente estabilizante, espessante, emulsificante; desta forma, podem ser aproveitadas na produção de diferentes produtos: bebidas, sopas, molhos, sobremesas, derivados de leite, biscoitos, massas e pães (CHO; DREHER, 2001). Muitos trabalhos têm sido conduzidos para aumentar a quantidade de fibras insolúveis nos produtos de panificação, biscoitos e barras de cereais com a adição de fibras de cereais, frutas, vegetais e celulose em pó (THEBAUDIN et al., 1997).

Além disso, pães, biscoitos, massas e salgadinhos parecem ser excelentes veículos de fibra alimentar; são produtos de boa aceitação, consumidos por todas as faixas etárias, e atingem principalmente idosos e crianças (GIUNTINI; LAJOLO; MENEZES, 2003).

A resolução RDC n 263 da Agência Nacional de Vigilância Sanitária define biscoito ou bolacha como os produtos obtidos pela mistura de farinha(s), amido(s) ou fécula(s) com outros ingredientes, submetidos a processos de amassamento e cocção, fermentados ou não. Podem apresentar cobertura, recheio, formato e textura diversos (BRASIL, 2005). O termo cookie, empregado nos Estados Unidos e na Inglaterra, pode ser considerado como sinônimo de biscoito (MANLEY, 1983)

A substituição de parte da farinha de trigo por resíduos da indústria de cerveja, farelo de arroz, fibra de milho, grãos destilados, sementes de girassol, farelo de trigo, farinha de jatobá, casca de batata e aveia tem sido relatada por vários autores na elaboração de cookies (ARTZ et al., 1990; ARORA; CAMIRE, 1994; CAMPBELL; KETELSEN; ANTENUCCI, 1994).

Este trabalho teve como objetivo elaborar cookies utilizando farinhas ricas em fibra alimentar (farinha de talo de couve e a farinha de talo de espinafre) visando boa aceitabilidade, menor custo e expressivo teor de fibras sem alterar as características sensoriais do produto final.

\section{Material e métodos}

\subsection{Material}

Foram adquiridos aproximadamente $13 \mathrm{~kg}$ de cada hortaliça do mesmo produtor, comercializadas na cidade do Rio de Janeiro.

As Farinhas de Talos (FT) foram obtidas a partir de talos de couve manteiga (Brassica oleracea, L.) e talos de espinafre (Spinacea oleracea, L.). Separaram-se os talos das folhas, os quais, posteriormente, foram higienizados em água corrente e sanitizados com hipoclorito de sódio a 200 ppm por 15 minutos.
Após essa etapa, as amostras foram cortadas em pequenos pedaços, branqueadas (em água fervente por 3 minutos) e escorridas. A secagem (desidratação) dos talos foi feita em estufa ventilada a $65{ }^{\circ} \mathrm{C}$ por 18 horas. Para a obtenção das farinhas, depois de desidratados, os talos foram triturados em liquidificador doméstico, acondicionados em frascos de vidro previamente esterilizados, selados e etiquetados, e estocados em freezer a $-18^{\circ} \mathrm{C}$.

Os demais gêneros alimentícios utilizados na formulação dos cookies (Tabela 1) foram adquiridos no mercado varejista local.

\subsection{Composição centesimal das Farinhas de Talo de Couve (FTC) e Talo de Espinafre (FTE)}

No Complexo Laboratorial do Instituto de Nutrição Josué de Castro, foram analisados os componentes das farinhas: umidade, cinzas, lipídios e proteínas (utilizando o fator de conversão de 5,7) segundo metodologia descrita pela AOAC (ASSOCIATION..., 1995), e fibra alimentar através do método descrito por van Soest (1963). A fibra alimentar analisada foi a Fibra Detergente Neutro (FDN) que corresponde ao somatório de celulose, hemicelulose e lignina, representando, portanto, o teor de fibra insolúvel. Os glicídios foram calculados por diferença das demais análises (NIFEXT). As análises de umidade e cinzas foram feitas em triplicata e as demais em duplicata.

\subsection{Formulação dos cookies}

Estudos preliminares demonstraram a inviabilidade de se utilizar níveis de $\mathrm{FT}$ acima de $15 \%$, por produzirem cookies com características sensoriais pouco aceitáveis.

Foram confeccionados 3 tipos de cookies (cookie controle, cookie com FTC e com FTE) por modificação da formulação padrão de sugar-sanap cookie, método 10-50D, descrito pela AACC (AMERICAN..., 1995). Nos cookies experimentais (FTC e FTE), substituiu-se a farinha de trigo total por $15 \%$ das farinhas de talos. As formulações estão apresentadas na Tabela 1.

A massa dos cookies foi processada manualmente da seguinte forma: formou-se um creme homogêneo com margarina e ovos. Em seguida, os ingredientes secos foram misturados e lhes foram adicionados $5 \mathrm{~mL}$ de água filtrada até obtenção de uma massa

Tabela 1. Formulação dos cookies.

\begin{tabular}{lccc}
\hline \multirow{2}{*}{ Ingredientes } & \multicolumn{3}{c}{ Tipo de formulação $(\mathrm{g} \%)$} \\
\cline { 2 - 4 } & Controle & Cookie $\mathrm{FTC}^{1}$ & Cookie $\mathrm{FTE}^{2}$ \\
\hline Farinha de trigo comercial & 47,5 & 33,4 & 33,4 \\
FTC $^{1}$ & - & 14,10 & - \\
FTE $^{2}$ & - & - & 14,10 \\
Açúcar mascavo & 13,0 & 13,0 & 13,0 \\
Açúcar refinado & 14,0 & 14,0 & 14,0 \\
Bicarbonato de sódio & 0,20 & 0,20 & 0,20 \\
Sal refinado & 0,30 & 0,30 & 0,30 \\
Margarina & 21,0 & 21,0 & 21,0 \\
Ovo & 4,0 & 4,0 & 4,0
\end{tabular}

${ }^{1}$ Farinha de Talo de Couve; ${ }^{2}$ Farinha de Talo de Espinafre. 
contínua. A massa foi moldada com o auxílio de uma forma circular e os cookies foram assados a $150-180^{\circ} \mathrm{C}$ por 20 minutos. Estes foram resfriados à temperatura ambiente e acondicionados em sacos de polipropileno e em vidros hermeticamente fechados para posteriores análises física, química e sensorial.

\subsection{Análises físicas e químicas e composição centesimal dos cookies}

As análises físicas dos cookies controle e experimentais (FTC e FTE) compreenderam os procedimentos descritos no macro método 10-50D da AACC (AMERICAN..., 1995) para determinação do peso, espessura, diâmetro, volume e densidade aparente antes e após a assadura. Os cookies foram pesados em balança digital marca CAZZA, modelo CA-110B, com capacidade máxima de $3 \mathrm{~kg}$ e graduação de $1 \mathrm{~g}$. A espessura e o diâmetro dos cookies foram determinados com régua de escala milimetrada. $\mathrm{O}$ volume foi calculado através da Equação 1:

$\mathrm{V}=\pi^{2}$

em que: $\mathrm{V}=$ volume/ $/ \pi=3,14 / \mathrm{R}=$ raio. A densidade foi calculada segundo adaptação do método de Ferreira (2002) através da Equação 2:

$\mathrm{D}_{\text {ap }}\left(\mathrm{g} \cdot \mathrm{cm}^{-3}\right)=\mathrm{M} / \mathrm{V}_{\text {ap }}$

em que: $\mathrm{D}_{\text {ap }}=$ densidade parente/ $\mathrm{M}=$ massa ou peso inicial da amostra/ $\mathrm{V}_{\mathrm{ap}}=$ volume aparente após assentamento da amostra. As análises foram conduzidas com 10 cookies provenientes de uma mesma fornada amostrados de forma aleatória assim que foram resfriados em temperatura ambiente.

A partir do peso pré e pós-cocção dos cookies também foi calculado o rendimento do produto pronto (pós-cocção) (ARAÚJO; GUERRA, 1992), segundo Equação 3:

Peso pós-cocção $\times$ 100/Peso pré-cocção

e o fator térmico segundo Equação 4:

Peso pós cocção/Peso pré-cocção

Para as análises químicas e determinação da composição centesimal, os cookies foram triturados em almofariz com pistilo. Foram feitas as seguintes análises químicas: a acidez total titulável e $\mathrm{pH}$. A primeira foi feita através de análise quantitativa e o $\mathrm{pH}$ foi determinado eletrometricamente por método descrito pelo Instituto Adolfo Lutz (SÃO PAULO, 1985), em triplicata, nos cookies crus (pré-cocção) e depois de prontos (pós-cocção).

Após esses procedimentos, os cookies foram submetidos à mesma determinação de composição centesimal feita nas farinhas de talos no Complexo Laboratorial do Instituto de Nutrição Josué de Castro: umidade, cinzas, lipídios e proteínas (utilizando o fator de conversão de 5,7) segundo metodologia descrita pela AOAC (ASSOCIATION..., 1995), e fibra alimentar através de dados obtidos da composição química das FT e Tabela de Composição de Alimentos de Mendez et al. (1995). Os glicídios foram calculados por diferença das demais análises
(NIFEXT). As análises de umidade e cinzas foram feitas em triplicata e as demais em duplicata.

\subsection{Análise de aceitação dos cookies}

A análise de aceitação foi realizada em amostras dos cookies experimentais (FTC e FTE), para os atributos: aspecto global, aroma, consistência e sabor. A metodologia utilizada foi escala hedônica estruturada de 9 pontos que abrange 9 - "gosto demasiadamente" a 1 - "recuso totalmente" aplicada à uma equipe composta por 100 provadores para ambos os cookies, incluindo visitantes e funcionários de um hospital militar. $\mathrm{O}$ teste de aceitação foi realizado em diferentes dias. Foi ofertado a cada provador uma unidade de cookie (aproximadamente $10 \mathrm{~g}$ ) embalado em papel alumínio à temperatura ambiente, além de uma Ficha de identificação e Ficha de teste de aceitação com a escala hedônica (Figura 1).

Este trabalho teve seu projeto submetido e aprovado pelo Comitê de Ética em Pesquisa (CEP) da Universidade Federal do Rio de Janeiro - UFRJ, sob o protocolo $n^{\circ}$ 127/07.

\subsection{Análise estatística}

Os dados obtidos foram avaliados pelo método de análise de variância (ANOVA) com comparação de médias pelo teste de Tukey em nível de confiança de 95\% e desvio padrão usando o software Statistical versão 6.0 (ARANGO, 2005).

\section{Resultados e discussão}

\subsection{Rendimento e composição química das Farinhas de Talos (FTC e FTE)}

O rendimento dos talos foram os seguintes: a partir de $13 \mathrm{~kg}$ de couve manteiga e de espinafre obtiveram-se 27 e 44,56\% de talos dos vegetais folhosos, respectivamente. Esses talos renderam 5,4\% de farinha de talo de couve e 3,8\% de farinha de talo de espinafre. $\mathrm{O}$ rendimento das farinhas foi reduzido em decorrência do alto teor de umidade dos talos in natura: $95,08 \%( \pm 0,16)$ para os talos de couve e $96,07 \%( \pm 0,22)$ para os talos de espinafre.

Os resultados médios de densidade energética e análise química das farinhas (FTC e FTE) estão apresentados na Tabela 2.

As FTC e FTE apresentaram teores de umidade compatíveis com produtos desidratados (MENDEZ et al.,1995; GOMES 2004) e também com farinhas conforme descrito pela Resolução RDC 263/2005 (BRASIL, 2005). Possuem alto teor de fibra alimentar e cinzas, baixo conteúdo de proteína e carboidratos disponíveis, quando comparados com hortaliças e frutos desidratados (MENDEZ et al.,1995). A quantidade de cinzas (10,80\% para FTC e $15,42 \%$ para FTE) foi consideravelmente maior do que a da farinha de milho, na qual Callegaro et al. (2005) encontraram 0,8\%, e também foi superior à encontrada na farinha de mandioca por Raupp et al. (1999), cujo valor foi 0,9\%.

Especificamente em relação ao teor de FDN, encontraramse $36,48 \%$ para FTC e $48,94 \%$ para FTE. Achados similares ao do presente estudo foram encontrados nas farinhas de talos 
elaboradas por Couto, Derivi e Mendez (2004), nos quais a farinha de talo de couve apresentou 33,95\% de fibra insolúvel $22,31 \%$ de celulose; $5,44 \%$ de hemicelulose; e 6,20\% de lignina, e a farinha de talo de espinafre apresentou 48,83-27,86\% de celulose; $10,71 \%$ de hemicelulose; e $10,26 \%$ de lignina.

Spiller e Shipley (1976 apud MAFFIA, 1991), apresentaram o conteúdo aproximado de FDN em alguns alimentos na matéria seca como: milho integral (13\%), aveia integral (31\%), farelo integral (45\%) e farelo de arroz (24\%). Quando comparados esses valores com os obtidos nas farinhas de talos (FTC - 36,48 e FTE - 48,94\%), notam-se valores próximos ao encontrado no farelo integral. No experimento de Ranzani et al. (1996), a farinha de casca de banana revelou ser importante fonte de fibra (FDN), correspondendo a cerca de 32\% do seu peso seco. Figuerola et al. (2005), por sua vez, analisou a concentração de fibra alimentar total nos resíduos industriais de polpa de maçã e de frutas cítricas produzidas na indústria de sucos (matéria seca), encontrando valores considerados elevados - entre 44,2 e $89,2 \%$. Assim sendo, pode-se dizer que as FT em estudo são ricas em fibra.

Ambas as farinhas apresentam baixa densidade energética, quando comparadas com outras, sendo enorme o potencial para elaboração de produtos alimentícios: farinha de mandioca rica em fibra insolúvel -197,6 kcal; farinha de folha de cenoura 293,56 kcal; e farinha de milho - 348 kcal (RAUPP et al., 1999; PEREIRA et al., 2003; CALLEGARO et al., 2005).

\subsection{Análises físicas e químicas e composição centesimal dos cookies}

Estão apresentados na Tabela 3 os resultados médios das análises físico-químicas realizadas com os cookies.

De acordo com a Tabela 3, dentre as análises físicas pré e pós-cocção, observamos que a maioria das determinações (peso, diâmetro, volume e densidade) não diferenciou estatisticamente

Ficha de identificação do consumidor

\begin{tabular}{|c|c|c|c|}
\hline \multicolumn{4}{|c|}{$\begin{array}{l}\text { Teste de preferência - Escala hedônica } \\
\text { Por favor, deguste e avalie a amostra marcando sua preferência com um "X" }\end{array}$} \\
\hline \multirow[t]{9}{*}{ Aspecto global } & Gosto demasiadamente & \multirow[t]{9}{*}{ Consistência } & Gosto demasiadamente \\
\hline & Gosto muito & & Gosto muito \\
\hline & Gosto moderadamente & & Gosto moderadamente \\
\hline & Gosto ligeiramente & & Gosto ligeiramente \\
\hline & Não gosto, nem degosto & & Não gosto, nem degosto \\
\hline & Desgosto ligeiramente & & Desgosto ligeiramente \\
\hline & Desgosto moderamente & & Desgosto moderamente \\
\hline & Desgosto muito & & Desgosto muito \\
\hline & Recuso totalmente & & Recuso totalmente \\
\hline \multirow[t]{9}{*}{ Aroma } & Gosto demasiadamente & \multirow[t]{9}{*}{ Sabor } & Gosto demasiadamente \\
\hline & Gosto muito & & Gosto muito \\
\hline & Gosto moderadamente & & Gosto moderadamente \\
\hline & Gosto ligeiramente & & Gosto ligeiramente \\
\hline & Não gosto, nem degosto & & Não gosto, nem degosto \\
\hline & Desgosto ligeiramente & & Desgosto ligeiramente \\
\hline & Desgosto moderamente & & Desgosto moderamente \\
\hline & Desgosto muito & & Desgosto muito \\
\hline & Recuso totalmente & & Recuso totalmente \\
\hline
\end{tabular}

Figura 1. Ficha de identificação do consumidor e teste de preferência. 
entre si ( $p>0,05)$, demonstrando, portanto, um elevado grau de similaridade entre os cookies experimentais, além da ocorrência de uma boa incorporação das FT. Houve diferença significativa $(\mathrm{p}<0,05)$ somente na determinação da espessura (pré e pós-cocção). Os cookies experimentais apresentaramse mais espessos em relação ao controle, destacando valores ainda maiores para o cookie FTC, isso ocorreu provavelmente devido à maior quantidade de materiais fibrosos presentes nos cookies experimentais. As fibras, por serem mais higroscópicas, retém água dando maior consistência à massa, evitando seu espalhamento, o que geralmente causa uma maior espessura (SILVA, M. R.; SILVA, M. P. A. P.; CHANG, 1998). Esse achado parece positivo, pois permite a confecção de um produto com maior espessura em relação ao convencional, sendo este um fator importante à sua comercialização. Igualmente ao observado neste trabalho, Silva, M. R., Silva, M. P. A. P. e Chang (1998) obtiveram

Tabela 2. Composição centesimal e densidade energética das farinhas de talos de couve (FTC) e de espinafre (FTE).

\begin{tabular}{lrr}
\hline \multirow{2}{*}{ Determinações $(\%)$} & \multicolumn{2}{c}{ Farinhas } \\
\cline { 2 - 3 } & \multicolumn{1}{c}{ FTC } & \multicolumn{1}{c}{ FTE } \\
\hline Umidade & $5,80^{\mathrm{b}}( \pm 0,45)$ & $44^{\mathrm{a}}( \pm 0,09)$ \\
Cinzas & $10,80^{\mathrm{a}}( \pm 0,08)$ & $15,42^{\mathrm{b}}( \pm 0,02)$ \\
Lipídios & $2,50^{\mathrm{a}}( \pm 0,85)$ & $2,25^{\mathrm{a}}( \pm 0,13)$ \\
Proteínas & $1,28^{\mathrm{a}}( \pm 0,05)$ & $1,51^{\mathrm{b}}( \pm 0,27)$ \\
Fibras (FDN) & $36,48^{\mathrm{a}}( \pm 0,30)$ & $48,94^{\mathrm{b}}( \pm 0,49)$ \\
Carboidratos & & $27,14( \pm 0,48)$ \\
Valor Energético $^{1}\left(\mathrm{kcal}^{2}\right)$ & $200,18( \pm 0,21)$ & $134,85( \pm 0,30)$ \\
\hline
\end{tabular}

Médias com letras iguais na horizontal não diferem significativamente entre si $(\mathrm{p}>0,05)$; 1 - NIFEXT/2 - kcal.100 $\mathrm{g}^{-1}$ do produto, segundo fatores de Atwater (MENDEZ et al., 1995) ${ }^{26}: 9$ kcal.g ${ }^{-1}$ de lipídio, 4 kcal.g ${ }^{-1}$ de proteína e 4 kcal.g ${ }^{-1}$ de carboidrato). uma maior espessura para as formulações adicionadas de farinha de jatobá, quando estas foram comparadas à formulação controle. Já Fasolin et al. (2007) constataram que as espessuras dos três tipos de cookies produzidos com farinha de banana verde não apresentaram diferenças significativas em relação ao cookie padrão.

Os valores de rendimento e fator térmico dos cookies formulados com as FT, apesar de mais elevados, não foram suficientes para demonstrarem diferença significativa em relação ao padrão. Assim como ocorreu no trabalho de Silva, Borges e Martins (2001), onde a adição de farinha de jatobá não afetou significantemente o rendimento dos cookies processados.

Quanto às análises químicas, ainda na Tabela 3, os cookies experimentais apresentaram valores mais altos $(\mathrm{p}<0,05)$ para a acidez titulável e valores mais baixos $(\mathrm{p}<0,05)$ para o $\mathrm{pH}$, quando comparados ao controle, tanto pré como póscocção. Comparando-se somente os cookies experimentais, também encontramos diferença $(\mathrm{p}<0,05)$ nos valores dessas determinações (pré e pós-cocção), sendo a acidez titulável maior no cookie FTC e o pH maior no cookie FTE. Pereira et al. (1999), ao elaborar biscoitos utilizando féculas fermentadas (araruta, batata baroa, batata inglesa, mandioca e polvilho azedo), encontrou valores semelhantes para acidez titulável nos biscoitos prontos, quando comparados aos valores encontrados nos biscoitos experimentais deste estudo (pré e pós-cocção), exceto o controle. Já os valores de $\mathrm{pH}$ encontrados por esses autores apresentaram-se inferiores aos encontrados em todas as amostras deste estudo (cookie controle, FTC e FTE), tanto pré como pós-cocção. Segundo Cereda (1987), durante a fermentação dessas féculas, ocorre um abaixamento do valor do $\mathrm{pH}$, com produção concomitante de ácidos orgânicos e compostos aromáticos. Santangelo (2006), ao elaborar panetones com farinha de semente de abóbora, encontrou

Tabela 3. Análises físicas e químicas dos cookies.

\begin{tabular}{|c|c|c|c|c|}
\hline \multicolumn{2}{|c|}{ Determinações } & \multicolumn{3}{|c|}{ Cookies } \\
\hline & & Controle & FTC & FTE \\
\hline \multirow[t]{2}{*}{ Peso (g) } & Pré-cocção & $10,0^{\mathrm{a}}( \pm 0,01)$ & $10^{\mathrm{a}}( \pm 0,01)$ & $10,0^{\mathrm{a}}( \pm 0,01)$ \\
\hline & Pós-cocção & $8,9^{\mathrm{a}}( \pm 0,74)$ & $9,2^{\mathrm{a}}( \pm 0,79)$ & $9,2^{\mathrm{a}}( \pm 0,63)$ \\
\hline \multirow[t]{2}{*}{ Espessura $(\mathrm{cm})$} & Pré-cocção & $0,52^{\mathrm{a}}( \pm 0,04)$ & $0,69^{c}( \pm 0,03)$ & $0,6^{\mathrm{b}}( \pm 0,01)$ \\
\hline & Pós-cocção & $0,51^{\mathrm{a}}( \pm 0,32)$ & $0,67^{c}( \pm 0,48)$ & $0,6^{\mathrm{b}}( \pm 0,01)$ \\
\hline \multirow[t]{2}{*}{ Diâmetro (cm) } & Pré-cocção & $3,54^{\mathrm{a}}( \pm 0,70)$ & $3,43^{\mathrm{a}}( \pm 0,18)$ & $3,45^{\mathrm{a}}( \pm 0,09)$ \\
\hline & Pós-cocção & $3,56^{\mathrm{a}}( \pm 0,09)$ & $3,58^{\mathrm{a}}( \pm 0,10)$ & $3,50^{\mathrm{a}}( \pm 0,11)$ \\
\hline \multirow[t]{2}{*}{ Volume $\left(\mathrm{cm}^{3}\right)$} & Pré-cocção & $30,96^{\mathrm{a}}( \pm 1,23)$ & $29,12^{\mathrm{a}}( \pm 2,90)$ & $29,41^{\mathrm{a}}( \pm 1,61)$ \\
\hline & Pós-cocção & $31,3^{\mathrm{a}}( \pm 1,69)$ & $31,65^{\mathrm{a}}( \pm 1,81)$ & $30,29^{\mathrm{a}}( \pm 1,95)$ \\
\hline \multirow[t]{2}{*}{ Densidade $\left(\mathrm{g} . \mathrm{cm}^{-3}\right)$} & Pré-cocção & $0,32^{\mathrm{a}}( \pm 0,09)$ & $0,35^{\mathrm{a}}( \pm 0,04)$ & $0,34^{\mathrm{a}}( \pm 0,02)$ \\
\hline & Pós-cocção & $0,29^{\mathrm{a}}( \pm 0,02)$ & $0,29^{\mathrm{a}}( \pm 0,03)$ & $0,31^{\mathrm{a}}( \pm 0,02)$ \\
\hline Rendimento (\%) & - & $89^{\mathrm{a}}( \pm 0,03)$ & $92^{\mathrm{a}}( \pm 0,18)$ & $92^{\mathrm{a}}( \pm 0,12)$ \\
\hline Fator térmico & - & $0,89^{\mathrm{a}}( \pm 0,02)$ & $0,92^{\mathrm{a}}( \pm 0,02)$ & $0,92^{\mathrm{a}}( \pm 0,03)$ \\
\hline \multirow[t]{2}{*}{ Acidez titulável } & Pré-cocção & $1,95^{\mathrm{a}}( \pm 0,20)$ & $4,01^{\mathrm{c}}( \pm 0,10)$ & $3.20^{\mathrm{b}}( \pm 0,18)$ \\
\hline & Pós-cocção & $1,34^{\mathrm{a}}( \pm 0,21)$ & $3,29^{c}( \pm 0,18)$ & $2,50^{\mathrm{b}}( \pm 0,25)$ \\
\hline \multirow[t]{2}{*}{$\mathrm{pH}$} & Pré-cocção & $6.89^{c}( \pm 0,07)$ & $6.43^{\mathrm{a}}( \pm 0,03)$ & $6.66^{\mathrm{b}}( \pm 0,03)$ \\
\hline & Pós-cocção & $6,73^{\mathrm{c}}( \pm 0,03)$ & $6.31^{\mathrm{a}}( \pm 0,01)$ & $6.42^{\mathrm{b}}( \pm 0,02)$ \\
\hline
\end{tabular}

Médias seguidas de letras iguais na horizontal não diferem significativamente entre si ( $\mathrm{p}>0,05)$. 
valores de pH (antes e após cocção) inferiores e valores de acidez titulável (antes e após cocção) superiores às médias encontradas nas amostras deste trabalho. Nos cookies em estudo, o desenvolvimento de compostos aromáticos durante o processamento térmico pode ter propiciado a reprodução dos eventos sob os valores de $\mathrm{pH}$ e acidez.

Em relação à composição centesimal dos cookies, a Tabela 4 mostra que a porcentagem de umidade dos experimentais foi maior $(\mathrm{p}<0,05)$ em relação ao controle, destacando valores mais elevados para o cookie FTE. Tais resultados estão de acordo com aqueles encontrados por Oliveira e Reyes (1990), Souza et al. (2000) e Silva, Borges e Martins (2001), os quais verificaram ter havido um incremento na umidade dos cookies à medida que se aumentou o teor de fibras, indicando que ocorreu uma maior retenção de água nos cookies, em virtude das características hidrofílicas da fibra.

O teor de cinzas foi maior $(\mathrm{p}<0,05)$ para o cookie FTE e de proteínas maior para o FTC. A porcentagem de carboidratos foi similar ( $p>0,05)$ entre os três. De fato, os achados mais expressivos observados nos cookies experimentais foram em relação aos baixos teores de lipídios e valor energético e ao elevado conteúdo de fibras alimentares, quando comparados à formulação padrão.

O teor de gorduras totais da formulação padrão foi de 14\%, enquanto que as formulações experimentais apresentaram aproximadamente $11 \%$ de lipídios, teores significativamente menores. Esses achados diferem dos encontrados por Fasolin et al. (2007), que não demonstraram diferença significativa no teor de extrato etéreo entre o biscoito padrão e biscoitos preparados com diferentes quantidades de farinha de banana verde (10, 20 e $30 \%)$.

Os cookies experimentais apresentaram densidade energética de $413,48 \mathrm{kcal} 100 \mathrm{~g}^{-1}$ do produto para FTC e $397,12 \mathrm{kcal} 100 \mathrm{~g}^{-1}$ do produto para FTE, teores significativamente menores que a formulação padrão (442,76 kcal. $100 \mathrm{~g}^{-1}$ do produto), destacando valores mais baixos para o FTE, certamente devido à sua maior porcentagem de fibras. Perez e Germani (2007) encontraram valores de 432,53 kcal.100 g ${ }^{-1}$ de cookies confeccionados com $15 \%$ de farinha de berinjela em substituição à farinha de trigo total, porcentagem igual à quantidade de FT utilizada nestes cookies, porém valor energético superior. Rodrigues et al. (2007) confeccionaram 3 tipos de cookies contendo café e obtiveram valores calóricos entre 498 e $502 \mathrm{kcal} 100 \mathrm{~g}^{-1}$ do produto, teores também superiores aos encontrados neste estudo. Além disso, as médias encontradas nas formulações com FT foram inferiores à maioria dos biscoitos fonte de fibras apresentados no Quadro 1, elaborado a partir de rótulos de produtos comerciais.

A quantidade de fibras foi maior nas formulações experimentais, quando comparadas à do controle. No Brasil, através da resolução ${ }^{\circ} 27$ da Agência Nacional de Vigilância Sanitária (BRASIL, 1998), estabelece-se que um alimento pode ser considerado fonte de fibra alimentar quando no produto acabado existir 3 g. $100 \mathrm{~g}^{-1}$ de fibras para alimentos sólidos e $1,5 \mathrm{~g} .100 \mathrm{~mL}^{-1}$ para alimentos líquidos, especificações estas atendidas pelos cookies experimentais em estudo. A adição de fibras alimentares em alimentos confere diferentes tipos de benefícios. Seu valor nutricional motiva consumidores a aumentar o consumo de fibras, que é aconselhado por nutricionistas. Podem também valorizar produtos agrícolas e subprodutos para utilizar como ingredientes (THEBAUDIN et al., 1997). Kissel, Prentice e Yamazaki (1975), ao elaborar cookies com substituição parcial de farinha de trigo por resíduo de cevada proveniente da indústria

Quadro 1. Quantidade de fibras e valor calórico dos cookies e de biscoitos comerciais* fontes de fibra alimentar total.

\begin{tabular}{|lcc|}
\hline Produto & $\begin{array}{c}\text { Quantidade de fibra alimentar } \\
\text { total (\%) }\end{array}$ & $\begin{array}{c}\text { Valor calórico em } \\
\text { kcal \% }\end{array}$ \\
\hline Cookie FTC & 3,37 & 413,48 \\
Cookie FTE & 4,25 & 397,12 \\
Biscoito A & 5,00 & 477,50 \\
Biscoito B & 6,10 & 476,09 \\
Biscoito C & 6,50 & 475,38 \\
Biscoito D & 3,08 & 453,85 \\
Biscoito E & 6,00 & 452,00 \\
Biscoito F & 5,00 & 446,67 \\
Biscoito G & 4,33 & 450,00 \\
Biscoito H & 3,53 & 417,65 \\
Biscoito I & 4,33 & 476,67 \\
\hline
\end{tabular}

*Os valores apresentados foram retirados dos rótulos dos biscoitos comerciais; A) Biscoito Vita life integral marca Nestlé São Luiz; B) Cookies aveia e coco marca Fibra Natus; C) Biscoito integral marca Jasmine; D) Bicoito Club Social Integral marca Nabisco; E) Biscoito salgado integral com gergelim marca Bauducco; F) Biscoito aveia e mel marca Nestlé; G) Cookies Integrais marca Vitao; H) Cookie Integral de Soja marca Good Soy; I) Cookies Integrais marca Barion.

Tabela 4. Composição centesimal e valor energético dos cookies.

\begin{tabular}{lrrr}
\hline Determinações (\%) & \multicolumn{1}{c}{ Cookies } \\
\cline { 2 - 4 } & \multicolumn{1}{c}{ Controle } & \multicolumn{1}{c}{ FTE } \\
\hline Umidade & $4,80^{\mathrm{a}}( \pm 0,12)$ & $5,68^{\mathrm{b}}( \pm 0,22)$ & $7,63^{\mathrm{c}}( \pm 0,13)$ \\
Cinzas & $1,06^{\mathrm{a}}( \pm 0,02)$ & $1,23^{\mathrm{a}}( \pm 0,32)$ & $2,54^{\mathrm{b}}( \pm 0,18)$ \\
Lipídios & $14,00^{\mathrm{b}}( \pm 0,49)$ & $10,92^{\mathrm{a}}( \pm 0,15)$ & $10,96^{\mathrm{a}}( \pm 0,46)$ \\
Proteínas & $0,50^{\mathrm{a}}( \pm 0,01)$ & $0,59^{\mathrm{b}}( \pm 0,01)$ & $0,52^{\mathrm{a}}( \pm 0,01)$ \\
Fibras (FDN) & $0,95^{\mathrm{a}}( \pm 0,01)$ & $3,37^{\mathrm{b}}( \pm 0,01)$ & $4,25^{\mathrm{c}}( \pm 0,01)$ \\
Carboidratos ${ }^{1}$ & $78,69^{\mathrm{a}}( \pm 0,04)$ & $78,21^{\mathrm{a}}( \pm 0,05)$ & $74,10^{\mathrm{a}}( \pm 0,05)$ \\
Valor energético $\left(\mathrm{kcal}^{2}\right)$ & $442,76^{\mathrm{c}}( \pm 0,51)$ & $413,48^{\mathrm{b}}( \pm 0,49)$ & $397,12^{\mathrm{a}}( \pm 0,32)$ \\
\hline
\end{tabular}

Médias com letras iguais na horizontal não diferem significativamente entre si (p > 0,05); ${ }^{1} \mathrm{NIFEXT} /{ }^{2} \mathrm{kcal} .100 \mathrm{~g}^{-1}$ do produto, segundo fatores de Atwater (MENDEZ et al., 1995$){ }^{26}$ : 9 kcal.g ${ }^{-1}$ de lipídio, 4 kcal.g ${ }^{-1}$ de proteína e 4 kcal.g-1 de carboidrato. 
cervejeira, encontraram níveis de 1,5 a 3,5\% de fibra alimentar. Vollendorf e Marlett (1994), por sua vez, analisaram o teor de fibra alimentar em cookies formulados com farinha de aveia e farinha de aveia e passas e obtiveram valores de 2,7 a 4,3\% (em base úmida). Silva (1997) encontrou níveis de 4,1 a 6,5\% de fibra alimentar total (em base seca) em cookies elaborados com farinha mista de trigo e jatobá. Protzek (1997) elaborou biscoitos com diferentes níveis de substituição de farinha de trigo por farinha de bagaço de maçã, encontrando valores de fibra alimentar total de 2,70 a 6,05\%. Já Perez e Germani (2007) encontraram valores de $8,22 \%$ (em base seca) em cookies confeccionados com 15\% de farinha de berinjela. Assim, comparando esses resultados com os do presente estudo, pode-se considerar que os cookies contendo FTC e FTE são boas fontes de fibra alimentar. No Quadro 1 está demonstrado o teor de fibras e o valor calórico dos cookies e de biscoitos comerciais considerados fontes dessa substância. Dentre os biscoitos industrializados, observa-se que todos possuem valor energético superior aos cookies em estudo, e em relação à quantidade de fibras, a maioria aponta valores próximos aos cookies FTC e FTE.

\subsection{Análise de aceitação dos cookies experimentais}

Analisando a Ficha de Identificação do Consumidor de ambos os cookies, indicamos algumas características da população de avaliadores. Essas características estão demonstradas na Figura 2.

A Figura 2 mostra uma população de avaliadores de $45,5 \%$ do sexo feminino e $54,5 \%$ do sexo masculino, predominantemente adulta, estando a maioria entre $20 \mathrm{e}$ 50 anos. O grau de escolaridade dominante foi o ensino médio e o local mais citado dentre os provadores para o consumo de biscoito doce foi em casa. Em relação à frequência de consumo alimentar, para a síntese de dados, os atributos "diário", "2 a $3 \times$ semana" e " $1 \times$ semana" foram reunidos em um único, correspondendo ao termo "semanal"; e os atributos "mensal", "raramente" e "nunca" foram agrupados no atributo "mensal". Neste caso, prevaleceu a frequência de consumo semanal tanto para o biscoito doce como para os outros alimentos pesquisados (refrigerante, biscoito salgado e leite). Esses resultados indicam, portanto, que estes produtos encontram-se frequentemente presentes na alimentação dos avaliadores.

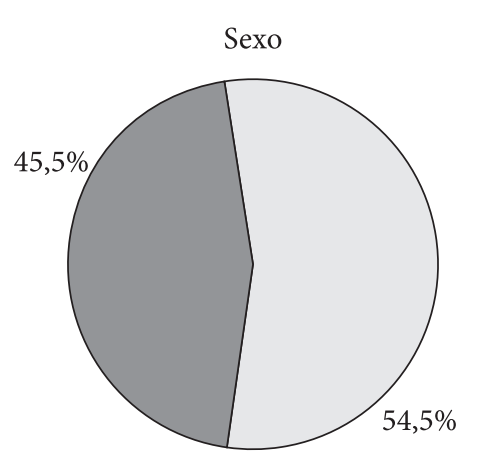

$\square$ Masculino $\square$ Feminino

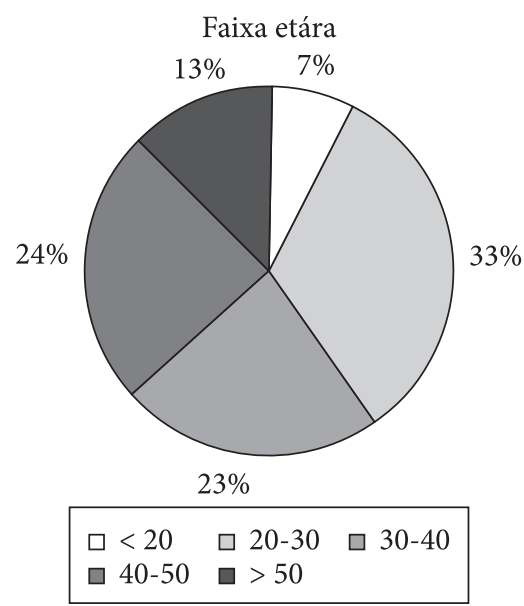

Local onde consome biscoito doce

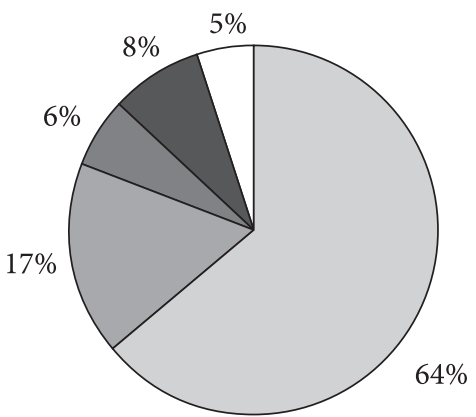

$\square$ Não marcou $\square$ Casa $\square$ Viagens $\square$ Lanchonetes/restaurantes $\square$ Outros
Grau de escolaridade

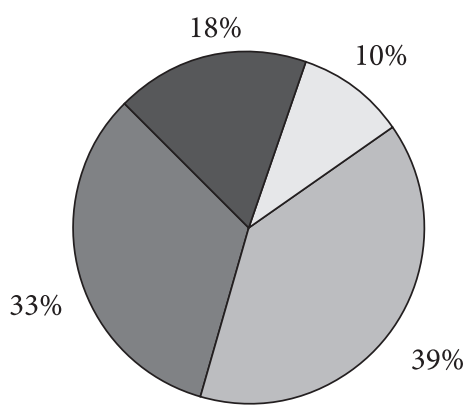

$\square$ Fundamental $\square$ Médio $\square$ Superior $\square$ Pós-graduação
Frequência de consumo alimentar

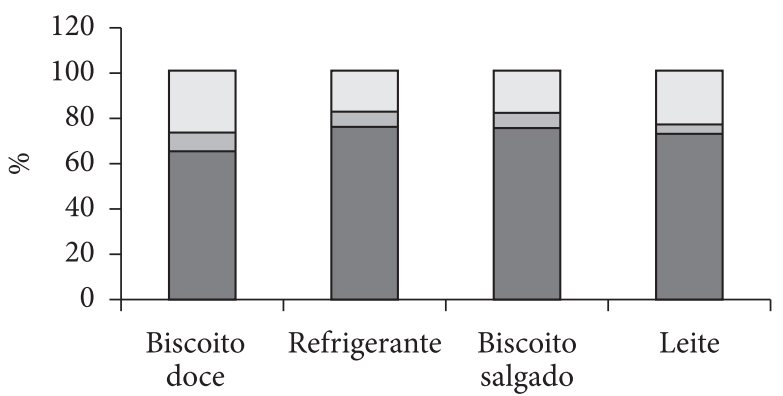

Alimentos

$\square$ Mensal $\square$ Quinzenal $\square$ Semanal

Figura 2. Perfil dos consumidores dos cookies FTC e FTE. 
As análises sensoriais das 2 formulações de cookies ricos em fibra alimentar estão demonstradas na Figura 3 e Tabela 5.

A Figura 3 demonstra a preferência dos provadores quanto aos 4 atributos avaliados nos cookies experimentais: aspecto global, aroma, consistência e sabor. Os valores hedônicos estão colocados de forma crescente, indo do valor 1 - correspondente ao termo hedônico "recuso totalmente" - até o valor 9 - correspondente ao termo hedônico "gosto demasiadamente". De uma maneira geral, pode-se dizer que mais de $65 \%$ dos provadores de ambos os cookies atribuíram notas acima de 5 (não gosto, nem desgosto) para todos os atributos. Nos atributos "aspecto global", "aroma" e "consistência” a nota prevalente foi 7 (gosto moderadamente) para ambos os cookies, no atributo "sabor" a nota prevalente foi 7 para FTC e 8 (gosto muito) para FTE.

Segundo Thebaudin et al. (1997), os principais critérios para aceitação de alimentos enriquecidos com fibras alimentares são: bom comportamento no processamento, boa estabilidade e aparência, e satisfação no aroma, na cor, na textura e na sensação deixada pelo alimento na boca. Certamente, de uma maneira geral, a maioria desses requisitos foram alcançados pelos cookies em questão. No trabalho de Possamai (2005), testes de análise sensorial feitos em pão de mel enriquecido com fibras do farelo de trigo e linhaça demonstraram que esses ingredientes não alteraram as características sensoriais e melhoraram a aceitabilidade do produto, tornando-o mais nutritivo. Já Cheung et al. (1998) concluíram que a adição de fibras afetou as propriedades sensoriais e a aceitabilidade dos cookies de chocolate, diferentemente desta pesquisa com cookies contendo FT, em que ambas as amostras obtiveram uma boa aceitabilidade entre os consumidores.

A Tabela 5 indica as médias das notas atribuídas às amostras de cookies, em relação aos 4 atributos avaliados. Os resultados elucidados nesta tabela estão de acordo com os apresentados na Figura 3.

Os valores de médias das 2 amostras indicam uma boa aceitação pelos provadores em relação aos 4 atributos analisados. Os atributos "aspecto global", "aroma" e "sabor" do cookie FTE apresentaram médias significantemente maiores $(p>0,05)$ em relação aos mesmos atributos do cookie FTC, indicando,

Tabela 5. Médias de aceitação da equipe de consumidores em relação ao Aspecto global, Aroma, Consistência e Sabor.

\begin{tabular}{ccrcc}
\hline Cookie & Aspecto global & Aroma & Consistência & Sabor \\
\hline FTC & $6,10^{\mathrm{a}}$ & $5,91^{\mathrm{a}}$ & $6,22^{\mathrm{a}}$ & $6,53^{\mathrm{a}}$ \\
FTE & $6,68^{\mathrm{b}}$ & $6,84^{\mathrm{b}}$ & $6,61^{\mathrm{a}}$ & $7,07^{\mathrm{b}}$ \\
\hline
\end{tabular}

Médias com letras iguais na coluna não diferem estatisticamente entre si $(p>0,05)$.

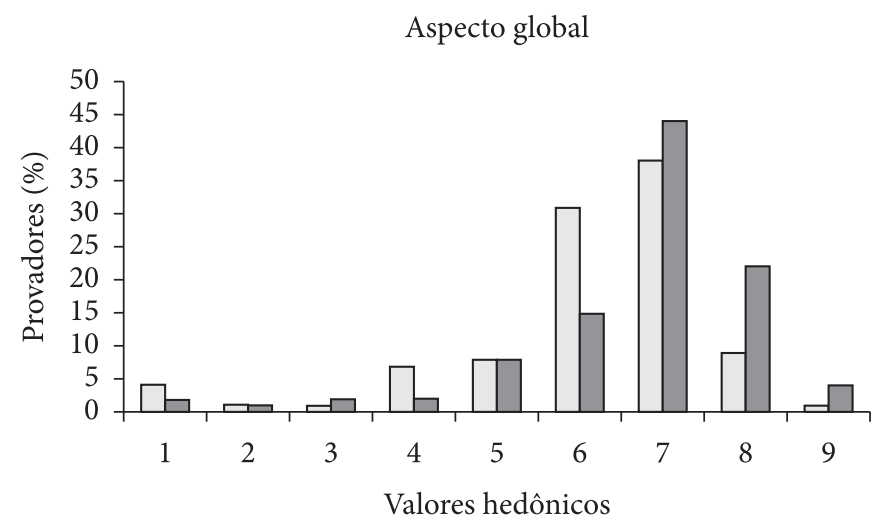

Consistência

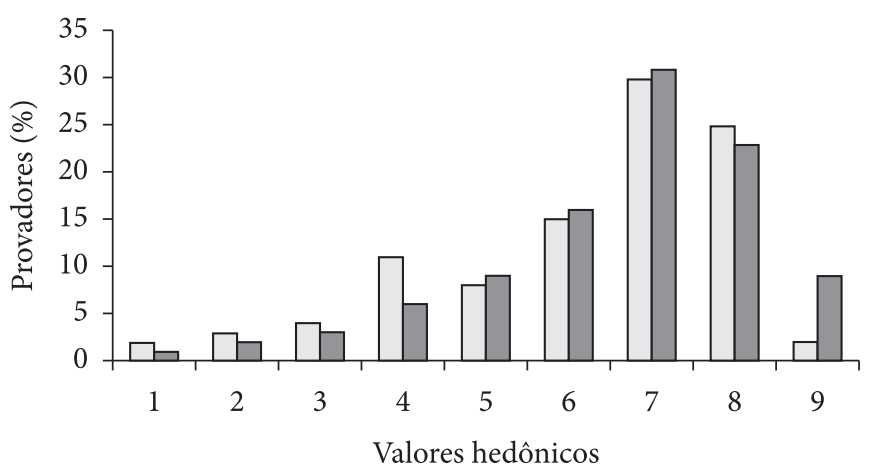

Aroma

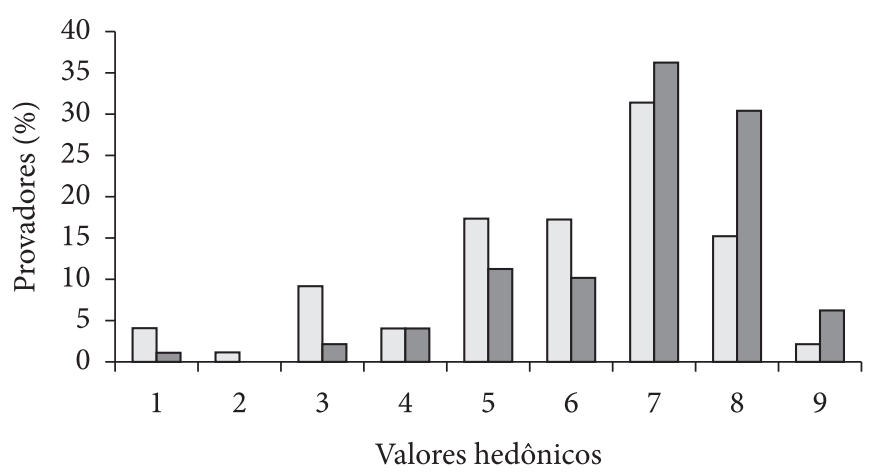

Sabor

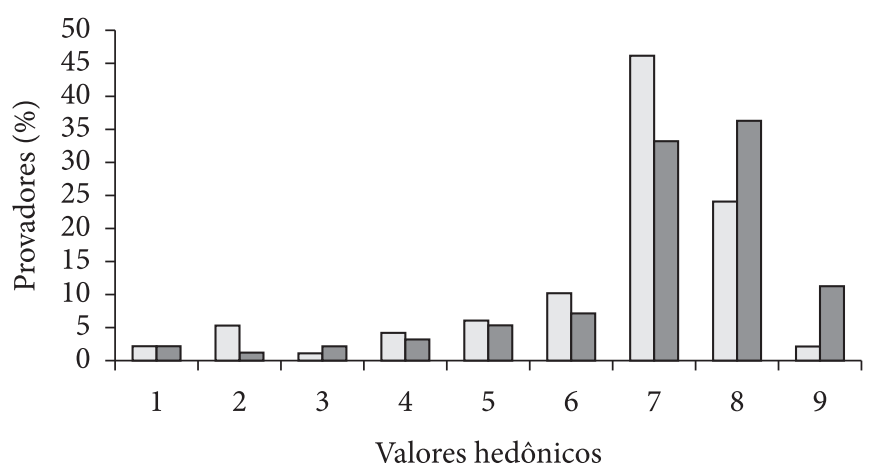

$\square$ FTC $\square$ FTE

Figura 3. Distribuição dos provadores quanto à preferência dos cookies elaborados com FTC e FTE para os atributos: Aspecto global, Aroma, Consistência e Sabor. 
portanto, um melhor desempenho do FTE do ponto de vista sensorial.

Moretti e Freitas (2006) encontraram boa preferência sensorial em barras de cereais com alto teor proteico e vitamínico, apontando médias semelhantes e até discretamente menores às encontradas nos cookies FTC e FTE em 2 quesitos "impressão global" e "sabor". Da mesma maneira, Battochio et al. (2006) concluíram que amostras comerciais de pães de forma integral foram bem aceitas pelos consumidores, sendo encontrados resultados bastante semelhantes aos deste estudo para os quesitos "sabor", "aroma" e "impressão global".

Segundo Fasolin et al. (2007), outros trabalhos realizados com diferentes tipos de biscoito têm demonstrado forte tendência das indústrias e pesquisadores em promover o enriquecimento de biscoitos, pois, por serem um produto de baixo custo podem facilmente ser consumidos pelas classes sociais menos privilegiadas.

\section{Conclusões}

As Farinhas de Talos (FT) utilizadas nos cookies experimentais possuem alto teor de fibra alimentar insolúvel e baixa densidade energética.

A maioria dos parâmetros físicos observados nos cookies experimentais foi similar ao padrão, com exceção somente da espessura (os cookies experimentais apresentaram-se mais espessos), demonstrando um elevado grau de similaridade entre os cookies e uma boa incorporação das FT.

Nas análises químicas, os cookies experimentais apresentaram valores mais altos para a acidez titulável e valores mais baixos para o $\mathrm{pH}$, quando comparados ao controle, provavelmente devido ao desenvolvimento de compostos aromáticos durante o processamento térmico destes.

Em relação à composição centesimal, observou-se menor taxa de gordura e densidade calórica e maior taxa de umidade e fibra nos cookies experimentais, que foram considerados fontes de fibras alimentares e demonstraram possuir densidade energética inferior a outros produtos comerciais fontes dessa substância.

Os resultados da análise sensorial demonstraram satisfatória aceitação dos cookies FTC e FTE quanto aos atributos: "aspecto global", "aroma", "consistência" e "sabor".

Portanto, os resultados apresentados neste trabalho indicam que as FT são matérias-primas de baixo custo e boas alternativas para a aplicação em produtos hipocalóricos ricos em fibras, com a possibilidade de adição dessas farinhas em substituição à quantidade total de farinha de trigo sem que haja perda da qualidade sensorial do produto.

\section{Agradecimentos}

À FAPERJ, pelo apoio financeiro ao projeto (Proc. E-26/171.167/2005), e às alunas de graduação em nutrição da UFRJ Annayra Silva de Rezende e Lívia da Silva Mattos, pelo auxílio em algumas etapas operacionais deste trabalho.

\section{Referências bibliográficas}

AMERICAN ASSOCIATION OF CEREAL CHEMISTS - AACC. Approved methods of the American Association of Cereal Chemists. 9. ed. Saint Paul: AACC, 1995. v. 1. paginação irregular.

ARANGO, H. G. Biostetatística: Teórica e Computacional. 2. ed. Rio de Janeiro: Guanabara Koogan, 2005. 423 p.

ARAÚJO, M. O. D.; GUERRA, I. M. M. Alimentos per capita. Natal: Universitária, 1992. $181 \mathrm{p}$.

ARORA, A.; CAMIRE, M. E. Performance of potato peels in muffins and cookies. Food Research International, v. 27, n. 1, p. 15-22, 1994.

ARTZ, W. E. Incorporation of corn fiber into sugar snap cookies. Cereal Chemistry, v. 67, n. 3, p. 303-05, 1990.

ASSOCIATION OF OFFICIAL ANALYTICAL CHEMISTS INTERNATIONAL - AOAC. Official methods of analysis Chemists. 15. ed. Washington, 1995. 1141 p.

BATTOCHIO, J. R. et al. Perfil Sensorial de pão de forma integral. Ciência e Tecnologia de Alimentos, v. 26, n. 2, p. 428-132, 2006.

BRASIL. Ministério da Saúde. Secretaria de Vigilância Sanitária. Portaria n 27, de 13 de janeiro de 1998. Aprova o Regulamento Técnico referente à Informação Nutricional Complementar (declarações relacionadas ao conteúdo de nutrientes), constantes do anexo desta Portaria. Diário Oficial União, Brasília, DF, 16 jan. 1998. seção 1.

BRASIL. Resolução RDC nº 263 de 22 de setembro de 2005. Aprova o Regulamento Técnico para produtos de cereais, amidos, farinhas e farelos, constantes do anexo desta Portaria. Diário Oficial União, Brasília, DF, 23 set. 2005.

CALlEGARO, M. G. K. et al. Determinação da fibra alimentar insolúvel, solúvel e total de produtos derivados do milho. Ciência e Tecnologia de Alimentos, v. 25, n. 2, p. 271-74, 2005.

CAMPBEll, L. A.; KeTElSEN, S. M.; ANTENUCCI, R. N. Formulating oatmeal cookies with calorie-sparing ingredients. Food Technology, v. 48, n. 5, p. 98-105, 1994.

CEREDA, M. P. Tecnologia e Qualidade do Polvilho Azedo. Informe Agropecuário, v. 13, n. 145, p. 63-68, 1987.

CHEUNG, L. et al. Nutritional value and acceptability of cookies with white wheat fiber, corn fiber and wheat bran. Food services management and quality management/outcomes research, v. 98, n. 9, p. A102, 1998. Resumo.

CHO, S. S.; DREHER, M. L. Handbook of Dietary Fiber. New York, NY: Marcel Dekker, Inc, 2001.

COUTO, S. R. M.; DERIVI, S. C. N.; MENDEZ, M. H. M. Utilização tecnológica de subprodutos da indústria de vegetais. Higiene alimentar, v. 18, n. 124, p. 12-22, 2004.

FASOLIN, L. H. et al. Biscoitos produzidos com farinha de banana: avaliações química, física e sensorial. Ciência e Tecnologia de Alimentos, v. 27, n. 3, p. 524-29, 2007.

FERREIRA, S. M. R. Controle da Qualidade em sistema de alimentação coletiva I. São Paulo: Varela, 2002. 173 p.

FIGUEROLA, F. et al. Fibre concentrates from Apple pomace and citrus peel as potential fibre sources for food enrichment. Food Chemistry, v. 91, n. 3, p. 395-401, 2005.

FOOD AND DRUG ADMINISTRATION - FDA. Center for Food Safety \& Applied Nutrition. A good labelling guide: appendix C Health Claims. [S.l.], 1998. Disponível em: <http://www.vm.cfsan. fda.gov>. Acesso em: 10 jan. 2007. 
GIUNTINI, E. B.; LAJOLO, F. M.; MENEZES, E. W. Potencial de fibra alimentar em países ibero-americanos: alimentos, produtos e resíduos. Archivos Latinoamericanos de Nutricion, v. 53, n. 1, p. 14-20, 2003.

GOMES, A. P. F. Avaliação de ratos diabéticos alimentados com sopa adicionada de goma guar. 2004. $81 \mathrm{f}$. Dissertação (Mestrado em Ciência e Tecnologia dos Alimentos)-Instituto de Tecnologia, Universidade Federal Rural do Rio de Janeiro, Seropédica, 2004.

KISSEL, L. T.; PRENTICE, N.; YAMAZAKI, W. T. Protein enrichment of cookie flours with wheat gluten and soy flour derivatives. Cereal Chemistry, v. 52, n. 6, p. 638-49, 1975.

MANLEY, D. J. R. Technology of biscuits: crackers and cookies. England: Ellis Horwood, 1983. 446 p.

MENDEZ, M. H. et al. Tabela de Composição de Alimentos. Niterói: EDUFF, 1995. $41 \mathrm{p}$.

MORETTI, R. H.; FREITAS, D. G. C. Caracterização e avaliação sensorial de barra de cereais funcional de alto teor protéico e vitamínico. Ciência e Tecnologia de Alimentos, v. 26, n. 2, p. 318-24, 2006.

OLIVEIRA, S. P.; REYES, F. G. R. Biscoito com alto teor de fibra de milho: preparo,caracterização química e tecnológica e teste de aceitabilidade. Ciência e Tecnologia de Alimentos, v. 10, n. 2, p. 273-86, 1990.

PENNA, E. W.; TUDESCA, M. V. Desarrollo de Alimentos. In: LAJOLO, F. M. et al. Fibra Dietética en Iberoamérica: tecnologia y Salu. Obtención, caracterización, efeito fisiológico y aplicación en alimentos. São Paulo: Varela, 2001. cap. 17, p. 245-54.

PEREIRA, G. I. S. et al. Avaliação química de folha de cenoura visando ao seu aproveitamento na alimentação humana. Ciência e Agrotecnologia, v. 27, n. 4, p. 852-57, 2003.

PEREIRA, J. et al. Féculas fermentadas na fabricação. de biscoitos: estudo de fontes alternativas. Ciência e Tecnologia de Alimentos, v. 19, n. 2, p. 287-93, 1999.

PEREZ, P. M. P.; GERMANI, R. Elaboração de biscoitos tipo salgado, com alto teor de fibra alimentar, utilizando farinha de berinjela (Solanum melongena, L.). Ciência e Tecnologia de Alimentos, v. 7, n. 1, p. 186-92, 2007.

POSSAMAI, T. N. Elaboração de pão de mel com fibra alimentar proveniente de diferentes grãos, sua caracterização físicoquímica, microbiológica e sensorial. 2005. 82 f. Dissertação (Mestrado em Tecnologia de Alimentos)-Universidade Federal do Paraná, Curitiba, 2005.

PROTZEK, E. C. Desenvolvimento de tecnologia para o aproveitamento do bagaço de maçã na elaboração de pães e biscoitos ricos em fibra alimentar. 1997. $94 \mathrm{f}$. Dissertação (Mestrado em Tecnologia Química)-Universidade Federal do Paraná, Curitiba, 1997.

RANZANI, T. C. et al. Avaliação química e biológica da casca de banana madura. Archivos Latinoamericanos Nutricion, v. 46, n. 4, p. 320-24, 1996.

RAUPP, D. S. et al. Composição e propriedades fisiológico-nutritivas de uma farinha rica em fibra insolúvel obtida do resíduo fibroso de fecularia de mandioca. Ciência e Tecnologia de Alimentos, v. 19, n. 3, p. 205-10, 1999.

RODRIGUES, M. A. A. et al. Desenvolvimento de formulações de biscoitos tipo cookie contendo café. Ciência e Tecnologia de Alimentos, v. 27, n. 1, p. 162-69, 2007.

SÁNCHEZ, J. L. R.; PÉREZ, M. F. Elaboración de productos alimentícios com fibra. La experiência em Cuba. In: LAJOLO, F. M. Fibra Dietética en Iberoamérica: Tecnologia y Salud: obtención, caracterización, efeito fisiológico y aplicación en alimentos. São Paulo: Varella, 2001. cap. 19, p. 263-265.

SANTANGELO, S. B. Utilização da farinha de semente de abóbora (cucurbita máxima) em panetone. 2006. 84 f. Dissertação (Mestrado em Ciência e Tecnologia de Alimentos)-Universidade Federal Rural do Rio de Janeiro, Seropédica, 2006.

SÃO PAULO. Secretaria de Estado de Saúde. Coordenadoria dos Serviços Técnicos Especializados. Instituto Adolfo Lutz. Normas analíticas do Instituto Adolfo Lutz: Métodos químicos e físicos para análise de alimentos. 3. ed. São Paulo: Instituto Adolfo Lutz, 1985. v. 1, 533 p.

SILVA, M. R. Caracterização química e nutricional da farinha de jatobá (Hymenaea stigonocarpa Mart.): desenvolvimento e otimização de produtos através de testes sensoriais afetivos. 1997. $154 \mathrm{f}$. Tese (Doutorado em Tecnologia de Alimentos)-Faculdade de Engenharia de Alimentos, Universidade Estadual de Campinas, Campinas, 1997.

SILVA, M. R.; BORGES, S.; MARTINS, K. A. Avaliação química, física e sensorial de biscoitos enriquecidos com farinha de jatobá-do-cerrado e de jatobá-da-mata como fonte de fibra alimentar. Brazilian Journal of Food Technology, v. 4, n. 73, p. 163-70, 2001.

SILVA, M. R.; SILVA, M. P. A. P.; CHANG, Y. K. Utilização da farinha de jatobá (Hymenaea stigonocarpa Mart.) na elaboração de biscoitos tipo cookie e avaliação de aceitação por testes sensoriais afetivos univariados e multivariados. Ciência e Tecnologia de Alimentos, v. 18, n. 1, p. 25-34, 1998.

SOUZA, M. L. et al. Processamento de cookies de castanha-do-brasil. In: CONGRESSO BRASILEIRO DE CIÊNCIA E TECNOLOGIA DE ALIMENTOS, 17., 2000, Fortaleza. Resumos... Campinas: SBCTA, 2000. v. 3, p. 1-12.

MAFFIA, V. C. C. Avaliação do farelo de arroz em substituição à farinha de trigo na panificação. Dissertação (Mestrado em Ciência e Tecnologia de Alimentos)-Universidade Federal de Visçosa, Visçosa, 1991.

THEBAUDIN, J. Y. et al. Dietary fibres: Nutritional and technological interest. Trends in Food Science Technology, v. 8, n. 2, p. 41-48, 1997.

van SOEST, P. J. Use of detergents in the analysis of fibrous feeds I- Preparation of fiber residues of low nitrogen. Journal of the Association Official Agricultural Chemists, v. 46, n. 5, p. 825-29, 1963.

VOLLENDORF, N. W.; MARLETT, J. A. Dietary fiber content and composition in home-prepared and commercially baked products: analysis and prediction. Cereal Chemistry, v. 17, n. 1, p. 99-105, 1994. 